\title{
Biokinetics of Radiolabeled Monoclonal Antibody BC8: Differences in Biodistribution and Dosimetry Among Hematologic Malignancies
}

\author{
Manuela Matesan ${ }^{1}$, Darrell R. Fisher ${ }^{1,2}$, Roger Wong ${ }^{1}$, Ajay K. Gopal ${ }^{3,4}$, Damian J. Green ${ }^{3,4}$, Brenda M. Sandmaier ${ }^{3,4}$, \\ William Bensinger ${ }^{5}$, John M. Pagel ${ }^{5}$, Johnnie Orozco ${ }^{3,4}$, Oliver W. Press ${ }^{\dagger 3,4}$, Ryan D. Cassaday ${ }^{3,4}$, Eric Hutchinson ${ }^{1}$, \\ Michelle Wanner ${ }^{1}$, Sujit Pal ${ }^{1}$, Carolyn Thostenson ${ }^{1}$, and Joseph G. Rajendran ${ }^{1}$ \\ ${ }^{1}$ Department of Radiology, University of Washington, Seattle, Washington; ${ }^{2}$ Versant Medical Physics and Radiation Safety, Richland, \\ Washington; ${ }^{3}$ Fred Hutchinson Cancer Research Center, Seattle, Washington; ${ }^{4}$ Department of Medicine, University of Washington \\ School of Medicine, Seattle, Washington; and ${ }^{5}$ Swedish Cancer Institute, Seattle, Washington
}

We reviewed ${ }^{111}$ In-DOTA-anti-CD45 antibody (BC8) imaging and bone marrow biopsy measurements to ascertain the biodistribution and biokinetics of the radiolabeled antibody and to investigate differences based on type of hematologic malignancy. Methods: Serial whole-body scintigraphic images (4 time points) were obtained after infusion of the ${ }^{111}$ In-DOTA-BC8 (176-406 MBq) into 52 adult patients with hematologic malignancies (lymphoma, multiple myeloma, acute myeloid leukemia, and myelodysplastic syndrome). Counts were obtained for the regions of interest for spleen, liver, kidneys, testicles (in men), and 2 marrow sites (acetabulum and sacrum), and correction for attenuation and background was made. Bone marrow biopsies were obtained 14-24 h after infusion, and the percentage of administered activity was determined. Absorbed radiation doses were calculated. Results: Initial uptake in liver averaged $32 \% \pm 8.4 \%$ (SD) of administered activity (52 patients), which cleared monoexponentially with a biologic half-time of $293 \pm 157 \mathrm{~h}$ (33 patients) or did not clear (19 patients). Initial uptake in spleen averaged $22 \% \pm 12 \%$ and cleared with a biologic half-time of $271 \pm$ $185 \mathrm{~h}$ (36 patients) or longer (6 patients). Initial uptake in kidney averaged $2.4 \% \pm 2.0 \%$ and cleared with a biologic half-time of $243 \pm 144 \mathrm{~h}$ (27 patients) or longer (9 patients). Initial uptake in red marrow averaged $23 \% \pm 11 \%$ and cleared with a biologic halftime of $215 \pm 107 \mathrm{~h}$ (43 patients) or longer (5 patients). Whole-body retention half-time averaged $198 \pm 75 \mathrm{~h}$. Splenic uptake was higher in the AML/MDS group than in the lymphoma group $(P \leq 0.05)$ or the multiple myeloma group $(P \leq 0.10)$. Liver represented the doselimiting organ. For liver uptake, no significant differences were observed among the 3 malignancy groups. Average calculated radiation absorbed doses per unit of administered activity for a therapy infusion of ${ }^{90} \mathrm{Y}$-DOTA-BC8 were $0.35 \pm 0.20 \mathrm{cGy} / \mathrm{MBq}$ for red marrow, $0.80 \pm 0.24 \mathrm{cGy} / \mathrm{MBq}$ for liver, $3.0 \pm 1.4 \mathrm{cGy} /$ $\mathrm{MBq}$ for spleen, $0.055 \pm 0.014 \mathrm{cGy} / \mathrm{MBq}$ for total body, $0.21 \pm$ $0.15 \mathrm{cGy} / \mathrm{MBq}$ for osteogenic cells, and $0.17 \pm 0.15 \mathrm{cGy} / \mathrm{MBq}$ for kidneys. Conclusion: ${ }^{111}$ In-DOTA-BC8 had a long retention time in liver, spleen, kidneys, and red marrow, and the highest absorbed doses were in spleen and liver. Few differences were observed by malignancy type. The exception was greater splenic

Received Aug. 30, 2019; revision accepted Jan. 3, 2020.

For correspondence or reprints contact: Manuela Matesan, Department of Radiology, University of Washington, Seattle, 1959 N.E. Pacific St., Seattle, WA 98195.

E-mail: mmatesan@u.washington.edu

†Deceased.

Published online Mar. 13, 2020.

COPYRIGHT (C 2020 by the Society of Nuclear Medicine and Molecular Imaging. uptake in the leukemia/MDS group than in the lymphoma or multiple myeloma group.

Key Words: BC8; anti-CD45; dosimetry; radioimmunotherapy

J Nucl Med 2020; 61:1300-1306

DOI: 10.2967/jnumed.119.234443

$\mathbf{R}$ adiolabeled monoclonal antibodies approved for treating patients with B-cell non-Hodgkin's lymphomas have shown both safety and efficacy. These radiopharmaceuticals include ibritumomab tiuxetan (Zevalin; Acrotech Biopharma), which is a ${ }^{90}$ Y-labeled antiCD20 monoclonal antibody (1), and tositumomab (Bexxar; GlaxoSmithKline), which is a ${ }^{131}$ I-labeled anti-CD20 monoclonal antibody (2,3). Since some lymphomas do not express CD20 antigens, the possibility of targeting other antigens has been investigated. Also, some lymphomas that express CD20 may have been modulated by prior extensive exposure to rituximab, therefore exhibiting a decreased response to radioimmunotherapy using antiCD20 antibodies (4). One such potential monoclonal antibody currently being assessed in clinical trials is $\mathrm{BC} 8$, a murine anti-CD45 IgG1 antibody (binding to all CD45 isoforms) that is conjugated with the DOTA chelate (also known as tetraxetan) for binding the radiotracers ${ }^{90} \mathrm{Y}$ and ${ }^{111} \mathrm{In}$. The CD45 antigen can be found on all hematopoietic cells except mature erythrocytes and platelets.

Both ${ }^{90}$ Y-DOTA-BC8 and ${ }^{131} \mathrm{I}-\mathrm{BC} 8$, directly labeled with iodine, were used in several clinical trials as part of the conditioning regimen before hematopoietic stem cell transplantation $(5-10)$. ${ }^{111}$ In-DOTABC8 was used as a low-activity tracer surrogate for ${ }^{90}$ Y-DOTA-BC8 to facilitate quantitative imaging for projecting required therapeutic doses.

We report here the biodistribution and dosimetry data obtained from 4 clinical trials using low tracer levels of ${ }^{111}$ In-DOTA-BC8 before high-dose ${ }^{90}$ Y-DOTA-BC8 for radioimmunotherapy. We assumed that both indium-labeled and yttrium-labeled conjugates behaved similarly in the same patient. We then looked for differences in radiolabeled antibody biodistribution among patients with different types of hematologic malignancies: lymphoma versus multiple myeloma and acute myeloid leukemia (AML)/myelodysplastic syndrome (MDS). We also looked at 2 potential differences in biodistribution using 2 different antibody BC8 concentrations (0.5 vs. $0.75 \mathrm{mg} / \mathrm{kg}$ ). 
TABLE 1

Clinical Protocol and Characteristics

\begin{tabular}{cclc}
\hline Protocol & Type & Patient population & Description \\
\hline 2728 & Phase I/II & B-NHL, T-NHL, HL & $\begin{array}{c}\text { 90Y-DOTA-BC8, BEAM chemotherapy, autologous peripheral blood stem } \\
\text { cell transplantation }\end{array}$ \\
\hline 2450 & Phase I & Multiple myeloma & $\begin{array}{c}90 \\
\text { 90-DOTA-BC8, fludarabine, TBI, HLA-matched blood stem cell transplantation }\end{array}$ \\
2468 & Phase I & AML, ALL, MDS & ${ }^{90}$ Y-DOTA-BC8, fludarabine, TBI (2 Gy), allogeneic stem cell transplantation \\
2361 & Phase I & B-NHL, T-NHL, or HL & ${ }^{90}$ Y-DOTA-BC8, autologous peripheral blood stem cell transplantation
\end{tabular}

B-NHL = B-cell non-Hodgkin's lymphoma; T-NHL = T-cell non-Hodgkin's lymphoma; $\mathrm{HL}=$ Hodgkin's lymphoma; BEAM = carmustine, etoposide, cytarabine, and melphalan; TBI = total-body irradiation; HLA = human leukocyte antigen; $A M L=$ acute myeloid leukemia; $\mathrm{ALL}=$ acute lymphoblastic leukemia.

\section{MATERIALS AND METHODS}

\section{Patient Population}

The clinical studies were approved by the Fred Hutchinson Cancer Research Center Institutional Review Board for protocols 2728, 2450, 2468 , and 2361, and written informed consent to participate was obtained from each patient.

Fifty-two patients with hematologic malignancies (lymphoma, multiple myeloma, AML, or MDS) from among 4 separate clinical trials were included in our analysis for biodistribution assessment and dosimetry. Of the 21 patients with lymphoma included in our analysis, there were 18 patients with aggressive forms of lymphoma ( 8 with diffuse large B-cell lymphoma, 1 with intermediate features between diffuse large B-cell lymphoma and Burkitt lymphoma, 4 with mantle cell lymphoma, 4 with Hodgkin's lymphoma, and 1 with peripheral T-cell lymphoma) and 3 patents with an indolent type of lymphoma (follicular lymphoma).

Radioimmunotherapy was added as part of the conditioning regimen before hematopoietic stem cell transplantation to reduce the side effects of classic high-dose conditioning therapy. Table 1 shows the patient population by clinical protocol. Patient demographics are summarized in Table 2.

TABLE 2

Patient Demographic Data

\begin{tabular}{|c|c|}
\hline Demographic & Total \\
\hline No. of patients & 52 \\
\hline Median age (y) & 55 (range, 26-76) \\
\hline \multicolumn{2}{|l|}{$\operatorname{Sex}(n)$} \\
\hline Female & 24 \\
\hline Male & 28 \\
\hline $\begin{array}{l}\text { Median }{ }^{111} \text { In-DOTA-BC8 } \\
\text { dose }(\mathrm{MBq})\end{array}$ & 329 (range, 176-406) \\
\hline \multicolumn{2}{|l|}{ Antibody concentration } \\
\hline $0.5 \mathrm{mg} / \mathrm{kg}$ & $\begin{array}{l}\text { Multiple myeloma } \\
\text { patients and AML/MDS } \\
\text { patients }\end{array}$ \\
\hline $0.75 \mathrm{mg} / \mathrm{kg}$ & Lymphoma patients \\
\hline $\begin{array}{l}\text { Patients with multiple } \\
\text { myeloma }(n)\end{array}$ & 15 \\
\hline Patients with AML/MDS $(n)$ & $11 \mathrm{AML} / 5 \mathrm{MDS}$ \\
\hline Lymphoma histology (n) & 21 \\
\hline
\end{tabular}

\section{Study Design}

The anti-CD45 mAb BC8 was produced in the Biologics Production Facility (Fred Hutchinson Cancer Research Center) in high purity under current good-manufacturing-practice conditions, as previously described (11). Patient serum was tested for the presence of human antimouse antibody using an enzyme-linked immunosorbent assay before infusion of ${ }^{111}$ In-DOTA-BC8 and ${ }^{90} \mathrm{Y}$-BC8-DOTA. Patients in two of the clinical trials (2450 [multiple myeloma] and 2468 [AML/ MDS]) received an antibody concentration (radiolabeled DOTABC8) of $0.5 \mathrm{mg} / \mathrm{kg}$. A protein concentration of $0.75 \mathrm{mg} / \mathrm{kg}$ was used for lymphoma patients (trials 2728 and 2361); this concentration was based on antibody dose-escalation results from a previous trial of ${ }^{131} \mathrm{I}-\mathrm{BC} 8$ in lymphoma that used a protein-escalation schema and sought to optimize the protein dose of BC8 (12).

${ }^{111}$ In-DOTA-BC8 (185-370 MBq [5-10 mCi]) was administered at the rate of $7.5 \mathrm{mg} / \mathrm{h}$ after premedication with acetaminophen, diphenhydramine, and hydrocortisone. To determine radiolabeled antibody biodistribution kinetics, 4-time-point whole-body planar anterior and posterior $\gamma$-camera images were obtained at the end of infusion (hour 0 ) and on the subsequent 3 d (usually 24, 48, and 72 or 120 h) using a Philips Brightview XCT camera with medium-energy collimators (Fig. 1). Acquired count data were corrected for attenuation and radioactive decay against a known counting standard. Organ volumes (liver, lungs, spleen, and kidney) were calculated from CT images for correcting the standard organ volumes $(13,14)$.

To determine the uptake and retention of radiolabeled antibody, regions of interest were drawn over the major imageable source organs, including 2 marrow sites (right or left acetabulum and sacrum), spleen, liver, and kidneys using the $180^{\circ}$ opposing-view quantitative planar technique as described by Eary et al. (15) and shown in Figure 2. Initial uptake and biologic retention half-times were determined from fitting mathematic functions (usually a single exponential) to the time-activity data. The best-fit functions were integrated to determine the time-integrated activity coefficients.

A bone marrow biopsy was obtained at approximately $24 \mathrm{~h}$ after the end of infusion, and the biopsy sample was weighed and counted against a weighed reference aliquot (counting standard) of the administered ${ }^{111}$ In activity to calculate the percentage administered activity per gram in marrow at the time of biopsy. The bone marrow biopsy results were then used as calibration points to quantitate the radioactivity observed in red marrow.

Images and bone marrow biopsy results obtained after ${ }^{111}$ In-DOTA$\mathrm{BC} 8$ administration were used to calculate radiation absorbed doses to normal organs and tissues, including marrow. For calculation, methods recommended by the special committee on Medical Internal Radiation Dose (MIRD) of the Society of Nuclear Medicine and Medical Imaging were applied to obtain radiation dose per ${ }^{90} \mathrm{Y}$ nuclear transformation for 


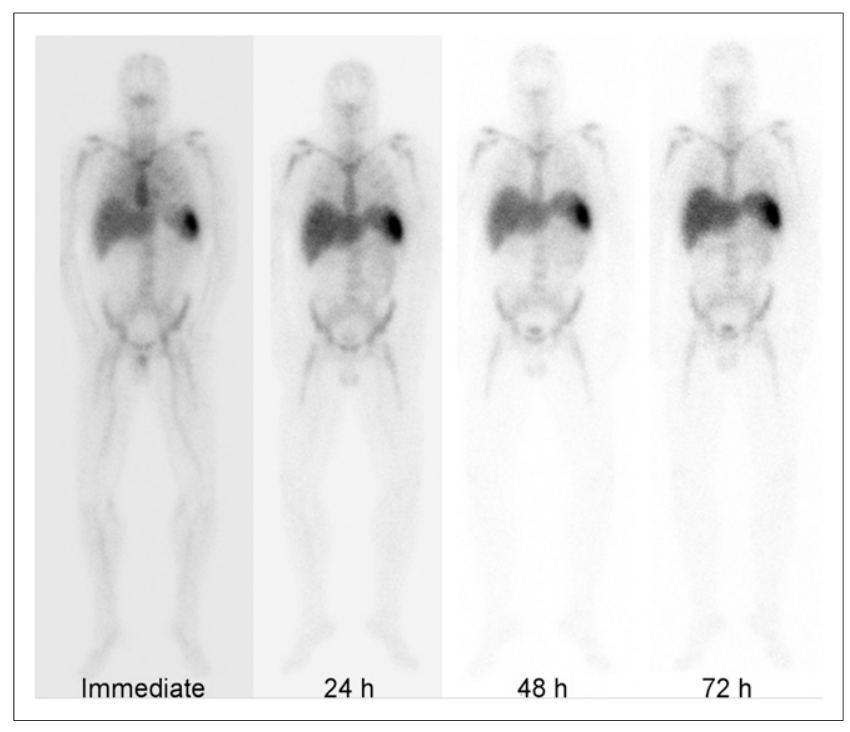

FIGURE 1. Whole-body anterior planar images of patient with B-cell non-Hodgkin's lymphoma after injection of low activity of ${ }^{111}$ In-DOTA-BC8 for dosimetric calculations before treatment with ${ }^{90}$ Y-DOTA-BC8.

each organ, including self-organ and cross-organ energy contributions and, ultimately, the absorbed dose to target organs of the body per unit of administered activity (cGy/MBq). Reported dosimetry for the marrow, spleen, liver, other nontarget organs, and the whole body delivered per $\mathrm{MBq}$ of ${ }^{90}$ Y-DOTA-BC8 (cGy/MBq) is presented in Table 3. Differences in biodistribution based on the type of disease were also assessed. Calculated therapy activity for ${ }^{90}$ Y-DOTA-BC8 was based on maximizing radiation dose to the critical limiting normal nontarget organ (usually liver) starting at $10 \mathrm{~Gy}$ for all patients to a level that would not exceed normal-organ toxicity, escalating the maximum normal-organ dose by 2 Gy per group. For example, for

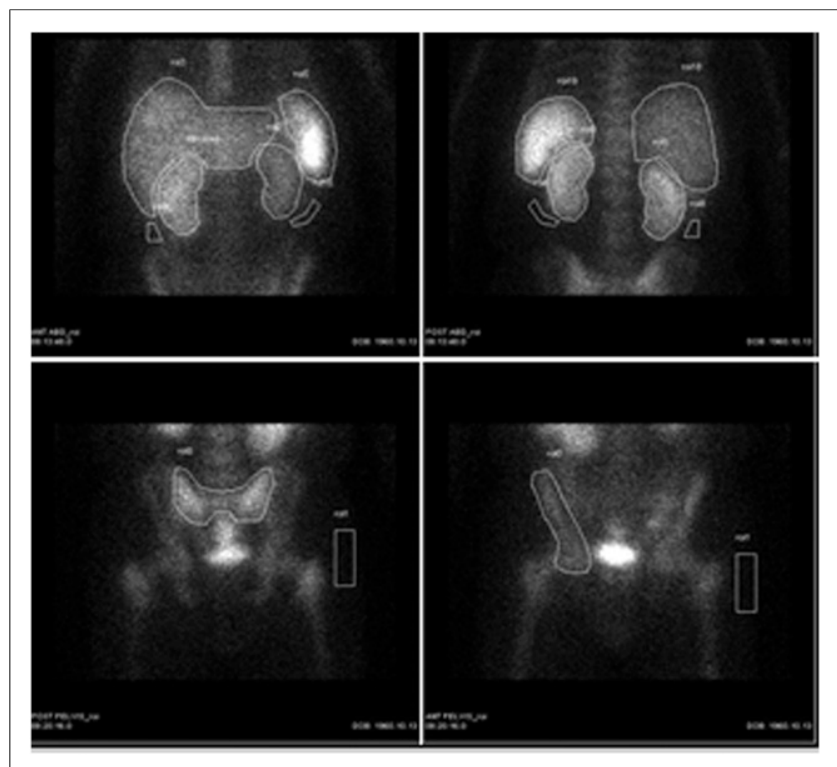

FIGURE 2. Imaging for dose calculation. Counts were obtained from regions of interest drawn over liver, spleen, and kidneys on anterior (A and $\mathrm{D})$ and posterior (B and $\mathrm{C}$ ) planar ${ }^{111} \mathrm{In}$-DOTA-BC8 images and used for dosimetry estimations together with counts per gram of tissue obtained from measurement of bone marrow biopsy specimen.
TABLE 3

Calculated Absorbed Doses per Unit of Administered Activity for ${ }^{90} \mathrm{Y}-\mathrm{DOTA}-\mathrm{BC} 8$ in Selected Target Organs

\begin{tabular}{lcc}
\hline \multicolumn{1}{c}{ Organ } & $\begin{array}{c}\text { Mean absorbed dose } \\
(\mathrm{cGy/MBq}) \pm \mathrm{SD}\end{array}$ & $\begin{array}{c}\text { Range } \\
(\mathrm{cGy} / \mathrm{MBq})\end{array}$ \\
\hline Bone marrow & $0.35 \pm 0.20$ & $0.007-0.745$ \\
\hline Spleen & $3 \pm 1.4$ & $0.026-8.83$ \\
Liver & $0.8 \pm 0.24$ & $0.375-1.7$ \\
Lung & $0.012 \pm 0.012$ & $0-0.035$ \\
Kidneys & $0.17 \pm 0.15$ & $0-0.6$ \\
\hline Osteogenic cells & $0.21 \pm 0.15$ & $0-0.594$ \\
Stomach & $0.0148 \pm 0.009$ & $0-0.035$ \\
\hline Small intestine & $0.012 \pm 0.009$ & $0-0.035$ \\
Large intestine & $0.012 \pm 0.009$ & $0-0.035$ \\
\hline Bladder wall & $0.012 \pm 0.009$ & $0-0.035$ \\
Total body & $0.055 \pm 0.014$ & $0.02-0.1$ \\
\hline
\end{tabular}

a multiple myeloma patient, the estimated dose to the liver (the dose-limiting organ), was $0.98 \mathrm{cGy} / \mathrm{MBq}(36.1 \mathrm{cGy} / \mathrm{mCi})$. For a desired therapy dose of 26 Gy to the dose-limiting organ, the calculated ${ }^{90} \mathrm{Y}$ activity was $2,650 \mathrm{MBq}(72 \mathrm{mCi})$.

We then looked at clinical outcomes for patients with high-risk lymphoma (8), AML/MDS (9), and multiple myeloma (10).

\section{RESULTS}

\section{Initial Uptake of ${ }^{111}$ In-Labeled-BC8}

Initial uptake of ${ }^{111}$ In-DOTA-BC8 in liver averaged $32 \% \pm$ $8.4 \%$ of administered activity ( 52 patients). The radioimmunoconjugate cleared from liver monoexponentially with a biologic halftime of $293 \pm 157 \mathrm{~h}$ (33 patients) or longer to infinity (19 patients). Initial uptake in spleen averaged $22 \% \pm 12 \%$ and cleared with a biologic half-time of $271 \pm 185 \mathrm{~h}$ (36 patients) or longer (6 patients). Initial uptake in kidney was $2.4 \% \pm 2.0 \%$ and cleared with a biologic half-time of $243 \pm 144 \mathrm{~h}$ (27 patients) or longer ( 9 patients). Initial uptake in red marrow was $23 \% \pm 11 \%$ and cleared with a biologic half-time of $215 \pm 107 \mathrm{~h}$ (43 patients) or longer (5 patients) (Fig. 3). Whole-body biologic retention half-times averaged $198 \pm$ $75 \mathrm{~h}$. The radiolabeled ${ }^{111}$ In-DOTA-BC8 was tenaciously retained in major source organs and the whole body. In some patients, no biologic clearance was observed.

\section{Radiation Absorbed Doses}

We calculated radiation absorbed doses per unit of administered activity of ${ }^{90}$ Y-DOTA-BC8 from analysis of the ${ }^{111}$ In-DOTA-BC8 serial planar imaging data and bone marrow radioactivity measurements: $0.35 \pm 0.20 \mathrm{cGy} / \mathrm{MBq}$ for red marrow, $0.80 \pm 0.24$ $\mathrm{cGy} / \mathrm{MBq}$ for liver, $3.0 \pm 1.4 \mathrm{cGy} / \mathrm{MBq}$ for spleen, $0.055 \pm 0.014$ cGy/MBq for total body, $0.21 \pm 0.15 \mathrm{cGy} / \mathrm{MBq}$ for osteogenic cells, and $0.17 \pm 0.15 \mathrm{cGy} / \mathrm{MBq}$ for kidneys (Table 3; Fig. 4).

\section{Differences Among Different Groups of Hematologic Diseases}

Initial Uptake. Further analysis of differences in biodistribution based on the patient's disease (lymphoma vs. multiple myeloma vs. AML/MDS) showed a statistical significantly higher initial splenic 


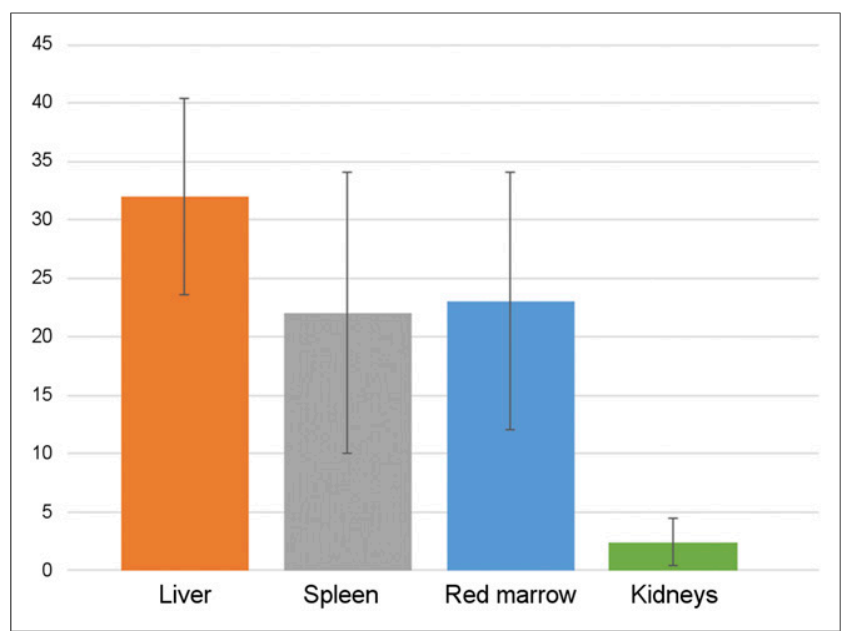

FIGURE 3. Chart illustrating initial percentage of administered activity taken up initially by the liver, spleen, red marrow, and kidneys.

uptake in the AML/MDS group than in the lymphoma group $(P \leq$ $0.05)$ or multiple myeloma group $(P \leq 0.1)$. At the $P \leq 0.10$ level of significance, we observed no significant differences in initial liver uptake or bone marrow uptake among the 3 groups (Fig. 5).

Radiation Absorbed Dose. For the liver, at the $P \leq 0.10$ level of significance, we did not observe significant differences between the multiple myeloma and lymphoma groups; however, we observed a lower liver radiation dose for AML/MDS patients than for multiple myeloma patients $(P \leq 0.1)$ or lymphoma patients $(P \leq 0.10)$ (Fig. 6$)$.

For the spleen, at the $P \leq 0.05$ level of significance, we observed differences between the multiple myeloma and lymphoma groups. At the $P \leq 0.10$ level of significance, we observed differences between the multiple myeloma and AML/MDS groups. However, we did not see significant differences between the AML/MDS and lymphoma groups $(P \leq 0.01)$ (Fig. 6).

For the red marrow, at the $P \leq 0.05$ level of significance, we did see significant differences between the AML/MDS and lymphoma groups.

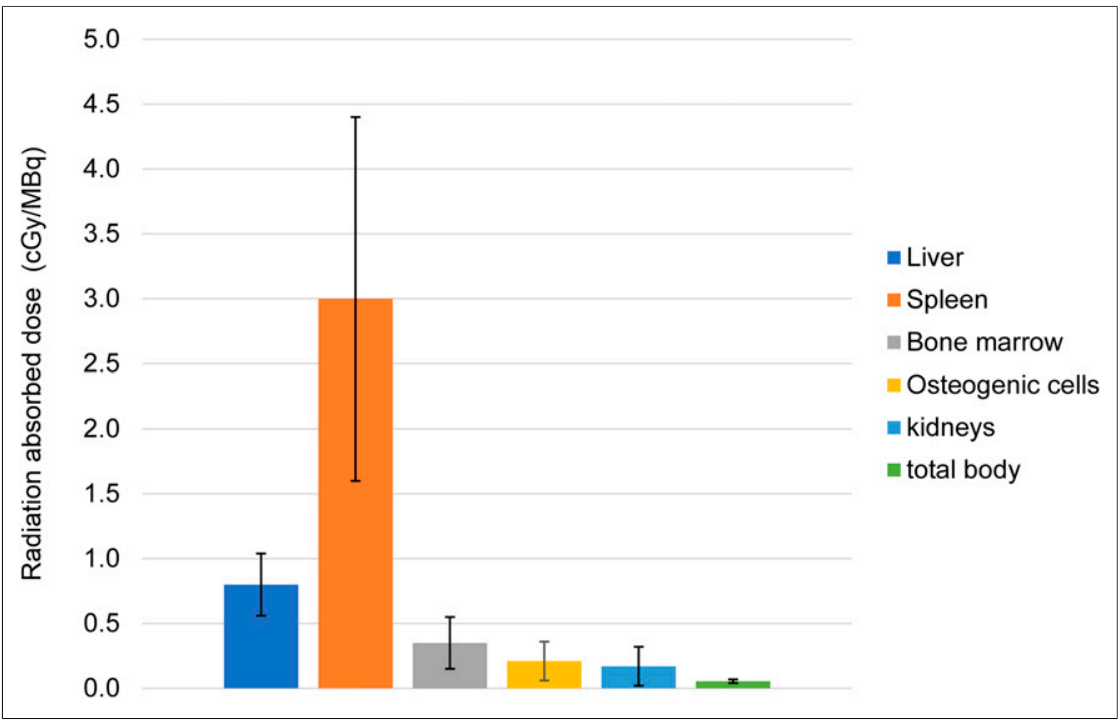

FIGURE 4. Absorbed doses per unit of administered activity (cumulative data for all groups of patients). Liver was dose-limiting normal organ in all patients; maximum dose to liver was 32 Gy for multiple myeloma and lymphoma groups and 28 Gy for AML/MDS group. No dose-limiting toxicities were observed.
We did not observe significant differences between the multiple myeloma and AML/MDS groups or between the multiple myeloma and lymphoma groups at the $P \leq 0.10$ level of significance (Fig. 6).

\section{Differences Based on Protein Concentration Administered}

We also compared the biodistribution of radiolabeled antibody in patients who received $0.5 \mathrm{mg} / \mathrm{kg}$ (multiple myeloma and AML/MDS groups) and patients who received $0.75 \mathrm{mg} / \mathrm{kg}$ (lymphoma group) (Figs. 7A and 7B). At the $P \leq 0.10$ level of significance, we did not observe differences in initial uptake by liver or marrow, but we did observe a greater splenic uptake for the group that received $0.5 \mathrm{mg} / \mathrm{kg}$ (multiple myeloma plus AML patients) than for the group that received $0.75 \mathrm{mg} / \mathrm{kg}$ (lymphoma patients).

Regarding the radiation absorbed dose at the $P \leq 0.05$ level of significance, we did find significant differences in protein concentration $(0.5$ vs. $0.75 \mathrm{mg} / \mathrm{kg})$ between dose to spleen and dose to marrow (cGy/MBq administered), but we found no significant differences at the $P \leq 0.10$ level for dose to liver. Therefore, protein concentration did not influence the liver dose.

\section{DISCUSSION}

Preclinical studies on the biodistribution of radiolabeled anti-CD45 were published previously (16). For example, the biodistribution of ${ }^{90}$ Y-labeled anti-CD45 antibody was demonstrated in a nonhuman primate model (macaques), a model that also predicted ${ }^{131}$ I-labeled anti-CD45 antibody biodistribution in humans (17). The results showed that the ${ }^{90}$ Y-labeled anti-CD45 antibody BC8 can deliver relatively selective radiation to hematopoietic tissues, with similar ratios of radiation delivered to target versus nontarget organs, as compared with the ${ }^{131}$ I-immunoconjugate in the same animal model. Another study, performed on mice, used $30 \mathrm{~F} 11$, an antibody with a slightly different structure (rat anti-CD45 antibody IgG2b) (18). Targeting with 30F11 also showed a favorable biodistribution, with effectively targeted bone, marrow, and spleen and minor uptake in nonhematologic organs. In humans, prior studies with anti-CD45 antibody were performed, either with a ${ }^{90}$ Y-conjugated rat $\mathrm{IgG} 2 \mathrm{a}$ monoclonal antibody (YAML568) or with a murine anti-CD45 IgG1 antibody (BC8).

Given that ${ }^{90} \mathrm{Y}$ is a pure $\beta$-emitting radioisotope, in our clinical studies a different radiotracer $\left({ }^{111} \mathrm{In}\right.$ bound to DOTA$\mathrm{BC} 8$ ) was used as a surrogate to determine the ${ }^{90}$ Y-antibody biodistributions needed to calculate internal radiation doses as previously performed with other radiolabeled antibodies $(19,20)$. After the dosimetry step was performed using ${ }^{111}$ In-DOTA-BC8, the patients received a treatment dose calculated by escalating the maximum normal organ doses by 2 Gy per group. The maximum dose received by the dose-limiting normal organ was set at 32 Gy for the multiple myeloma group (14 patients) and the lymphoma group (19 patients) and 28 Gy for the AML/MDS group (15 patients) (21). No dose-limiting toxicities were observed. One patient from the multiple myeloma group and 1 from the AML/MDS group did not receive treatment because human antimouse antibodies developed after the tracer (low-level)-labeled 


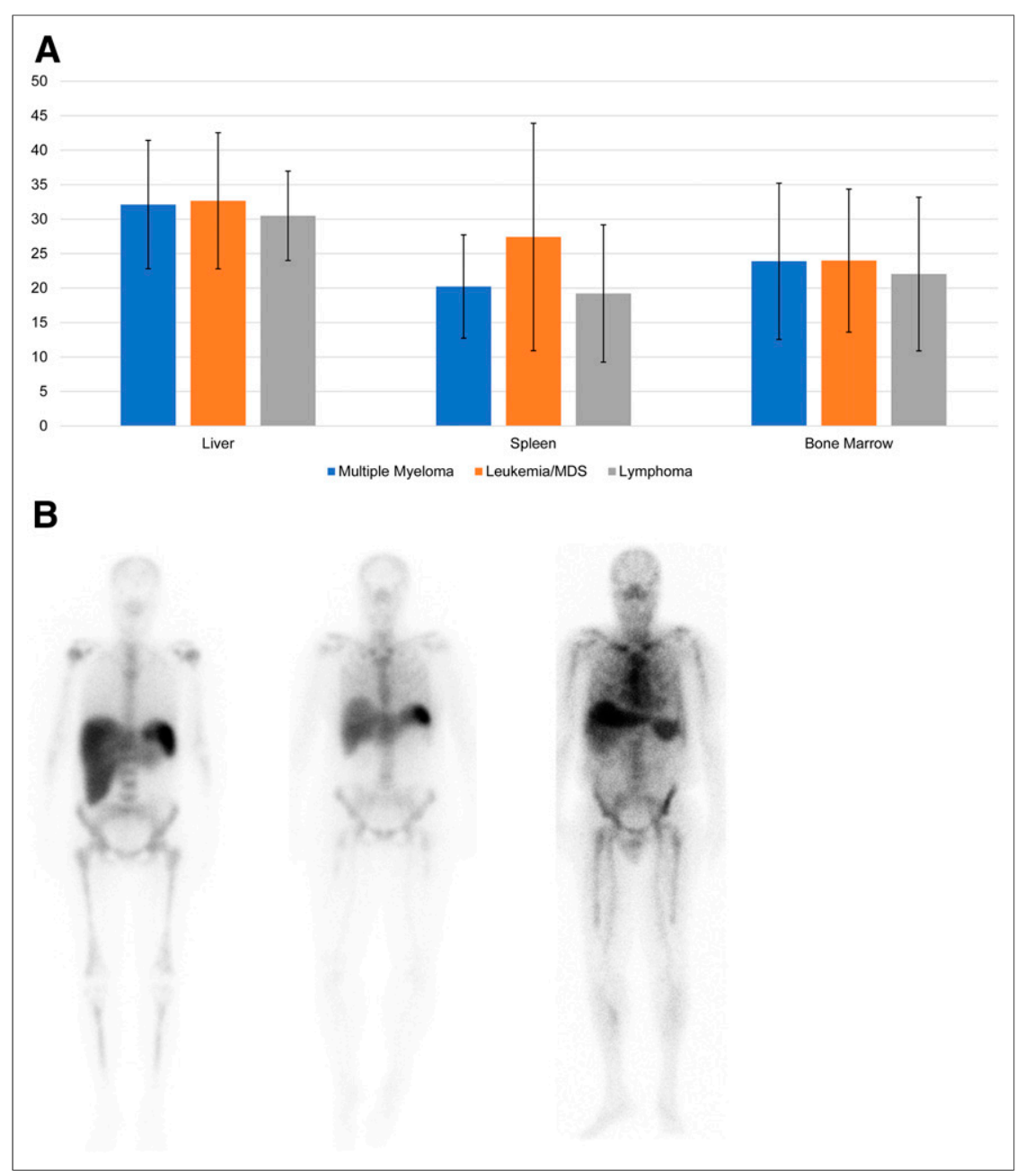

FIGURE 5. (A) Percentage of initial uptake for patient groups. Initial splenic uptake was higher in AML/MDS group than in lymphoma group $(P \leq 0.05)$ or multiple myeloma group $(P \leq 0.1)$. No significant intergroup differences were observed for initial liver uptake or bone marrow uptake $(P \leq 0.10)$. (B) Anterior whole-body images obtained immediately after ${ }^{111}$ In-DOTA-BC8 infusion (time 0: initial uptake) in 3 different patients: 1 with $\mathrm{AML}(\mathrm{A}), 1$ with multiple myeloma (B), and 1 with diffuse large B-cell lymphoma (C). Although for liver there were no significant differences in initial uptake, lower radiation dose (at $P \leq 0.1$ ) was found for AML/MDS group than for other 2 groups (probably because radiation absorbed dose is inversely proportional to mass).

imaging dose of ${ }^{111} \mathrm{In}-\mathrm{BC} 8$ was given. One subject from the lymphoma group had grade 4 hypotension during and after infusion.

Dosimetry showed that the radiolabeled antibody ( ${ }^{111} \mathrm{In}$-DOTABC8) was retained with a very long or infinitely long biologic retention half-time (corrected for physical decay of ${ }^{111} \mathrm{In}$ ) in liver, spleen, red marrow, and kidneys, with the highest doses being in spleen and liver. The long biologic retention half-times (approaching 10 days and longer) resulted in a more efficient delivery of radiation to the patient, because decay occurred in vivo rather than after excretion. Long biologic retention times were common to all patients from the 4 studies included in this analysis. The reason for this behavior is unknown but is probably due to the fact that the ${ }^{111} \mathrm{In}$ remains conjugated to the antibody and is not metabolized or catabolized. This finding was not seen in the animal model when AC8 or $30 \mathrm{~F} 11$ was used, nor was it seen in clinical studies when biodistribution of radiolabeled anti-CD20 antibody ( ${ }^{111}$ In-ibritumomab) was determined $(20,22,23)$ or when radiolabeled andi-CD45 antibody ${ }^{131} \mathrm{I}-\mathrm{BC} 8$ or YAML568 dosimetric calculations were made $(24,25)$. We could achieve desired doses to the nontarget limiting organ (liver in all patients) of 32 Gy for multiple myeloma patients and 28 Gy for AML/MDS and lymphoma patients without dose-limiting toxicity seen. Also, our results showed a higher calculated radiation dose to liver than to bone marrow-a finding that was not seen on preclinical studies or clinical studies that used ${ }^{111} \mathrm{I}-\mathrm{BC} 8$ or YAML568. For instance, when ${ }^{90} \mathrm{Y}-\mathrm{AC} 8$ antibody was used in preclinical studies, the delivered estimated radiation absorbed doses were 5-7 times higher to spleen and 2-4 times higher to marrow than to lungs or liver (17). However, the murine antihuman antibody used (BC8) has a structure that differs from the murine anti-CD45 antibody, called 30F11, and the nonhuman primate anti-CD45 called AC8. This difference might be explained by the differences in antibody structure or in species (18). Another potential explanation is the changes in biodistribution that are known to occur when cold antibody is not used before administration of the labeled one. Addition of supplemental unlabeled antibodies was shown to be necessary to achieve optimal biodistribution when another anti-CD45 monoclonal antibody with a structure different from $\mathrm{BC} 8$, called YAML568, was used (26). However, the unlabeled antibodies were not given in these 4 clinical trials because, in humans, prior biodistribution studies using ${ }^{131} \mathrm{I}-\mathrm{BC} 8$ without a preloading dose of unlabeled antibodies showed a favorable distribution, with the highest dose being to bone marrow and spleen, followed by the normal organs (liver was the highest doselimiting normal organ) $(24,25)$.

Since the patients with multiple myeloma and AML/MDS received a $0.5 \mathrm{mg} / \mathrm{kg}$ amount of protein and lymphoma patients received $0.75 \mathrm{mg} / \mathrm{kg}$, we also analyzed differences in biodistribution based on the protein concentration. Comparison of the initial uptake and radiation absorbed dose to liver between these 2 groups ( 0.5 vs. $0.75 \mathrm{mg} / \mathrm{kg}$ ) did not show a statistically significant difference in liver initial uptake or (average) liver-absorbed radiation dose. This information is important because liver was the doselimiting organ in all 3 groups of patients and because the radiation dose to liver was therefore used to calculate the treatment dose.

Initial uptake in spleen was higher for the multiple myeloma and AML/MDS groups (which received $0.5 \mathrm{mg} / \mathrm{kg}$ ) than for the lymphoma group $(0.75 \mathrm{mg} / \mathrm{kg})$. It is likely that increasing the amount (mg) of protein in lymphoma patients might have increased splenic uptake (since higher protein improved tumor dosimetry (12)); however, it did not reach the level observed in patients with leukemia where there is a significant increase in cellularity due to tumor cell infiltration.

Differences in biodistribution based on different groups of disease were also found. There was higher initial splenic uptake in leukemia patients than in lymphoma or multiple myeloma patients, probably because there was also a high percentage of patients with enlarged 


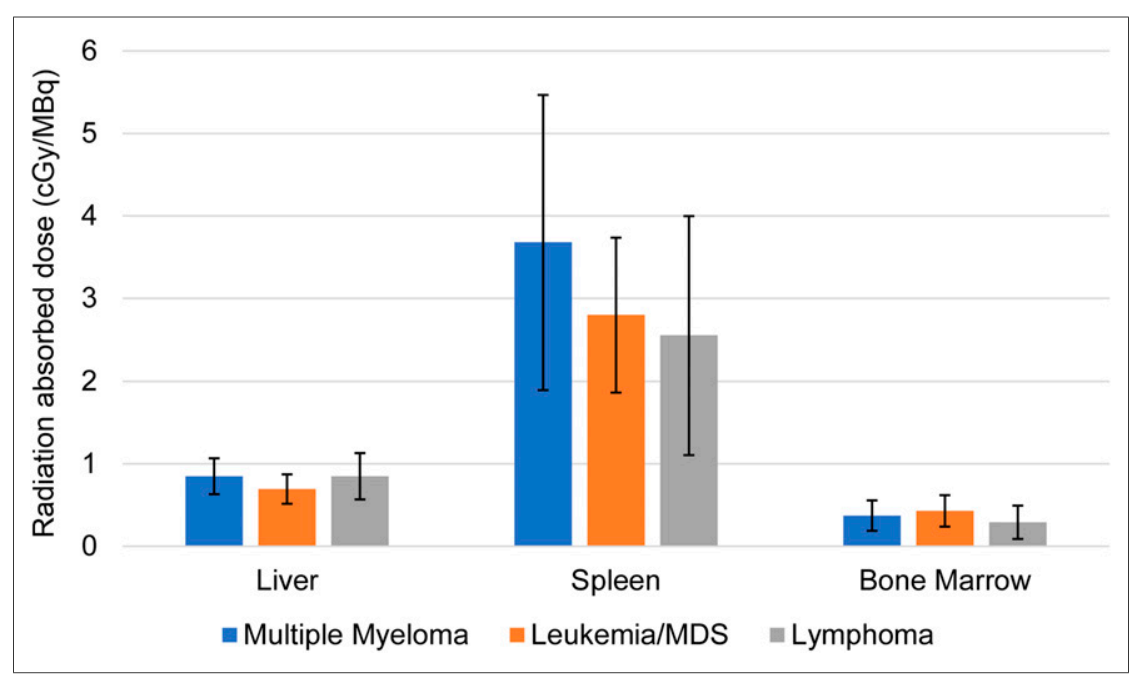

FIGURE 6. Differences in radiation absorbed dose among groups. In spleen, dose was higher for multiple myeloma group than for lymphoma group $(P \leq 0.05)$, and in bone marrow, dose was higher for AML/MDS group than for lymphoma group $(P \leq 0.05)$.

spleen due to disease infiltration. In addition, the number of target sites is greater in spleen of leukemia patients. Even though the amount of $\mathrm{BC} 8$ protein was greater in lymphoma patients than in leukemia patients, the overriding factor appears to be the greater number of binding sites in the leukemia patient spleen. Thus, the effect of a higher number of target sites in spleens of leukemia patients is stronger than merely the milligrams of protein administered. However, when the radiation absorbed dose in spleen was calculated, it was higher in multiple myeloma patients than in AML/MDS or lymphoma patients, likely because radiation absorbed dose is inversely proportional to mass.

Radiation absorbed dose in liver was higher for multiple myeloma patients than for AML/MDS patients. Similarly, for bone marrow there was a higher radiation absorbed dose in AML/ MDS patients than in lymphoma patients.

For patients treated with ${ }^{111}$ In-DOTA-BC8, our group previously published data on the influence of splenic size on the bone marrow dose received. Increasing spleen size reduced bone marrow dose, but measurement uncertainties associated with the marrow content of biopsy specimens may have reduced the correlation coefficient $(r=0.25)$. The spleen acts as a natural sink for radiolabeled antibody, reducing uptake and retention of radiolabeled antibody in red marrow (27). Other authors have also discussed the possibility that spleen has a role in antibody biodistribution (28).

\section{CONCLUSION}

Compared with other radiolabeled antiCD45 antibodies, ${ }^{111}$ In-DOTA-BC8 was retained longer in liver, spleen, red marrow, and kidneys, with the highest doses being in spleen and liver. These long biologic half-times (approaching 10 days and longer) result in a more efficient delivery of radiation to the patient because decay takes place in vivo rather than after excretion. This pattern of biodistribution was not seen on preclinical or clinical studies that targeted CD45 (using other radiolabeled antibodies) and might be partially due to DOTA chelator, which is present in the structure of this radiolabeled antibody.

Liver, having the highest calculated radiation absorbed dose after spleen, represented the critical nontarget organ for all patient cases and was the designated limiting normal organ for determining administered radioactivity. This finding might have been related to the DOTA chelator presence and the fact that, in these studies, a cold antibody pretreatment was not given. Regarding the protein concentration $(0.5$ vs. $0.75 \mathrm{mg} / \mathrm{kg})$, we did not observe significant differences in radiation absorbed dose to the liver (at $P \leq 0.10$ ). Therefore, protein concentration did not influence liver absorbed dose.

Few differences in ${ }^{111}$ In-DOTA-BC 8 biodistribution were observed by malignancy type. The exception was greater initial splenic uptake in the AML/MDS group than in the lymphoma or multiple myeloma groups.

\section{DISCLOSURE}

This work is supported by program project grant P01 CA 44991, and the grant principal investigator is Oliver Press, MD,
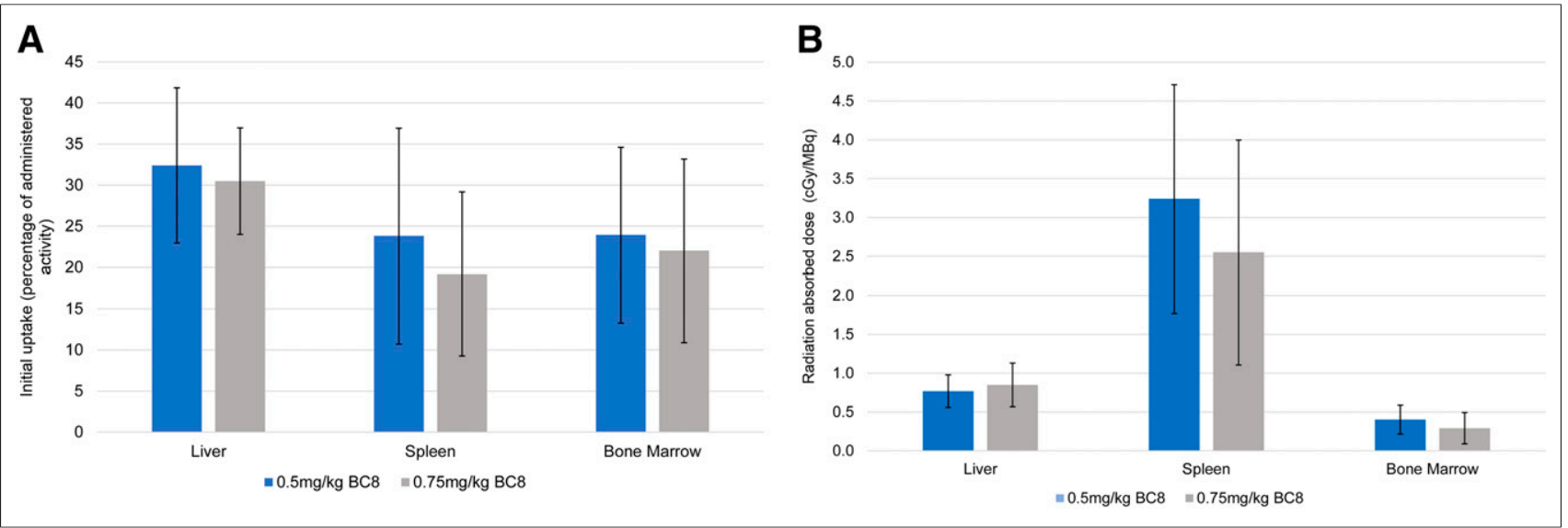

FIGURE 7. Differences in initial uptake and radiation absorbed dose based on protein concentration ( $0.5 \mathrm{vs}$. $0.75 \mathrm{mg} / \mathrm{kg})$. (A) Statistical significance was not reached $(P>0.05)$ for differences in initial uptake in liver, spleen, or bone marrow. (B) No significant differences based on protein concentration were obtained for radiation dose absorbed by liver (which was critical limiting nontarget organ for all patients). 
$\mathrm{PhD}$. This work is also supported by $\mathrm{R} 21$ grant CA155911, and Damian J. Green, MD, is the principal investigator. Darrell Fisher is an employee of Versant Medical Physics and Radiation Safety, which provides contract professional services for various clients, including radiopharmaceutical suppliers and others involved in development and testing of diagnostic and therapeutic medical devices. Ryan Cassaday received research funding from Amgen, Kite/Gilead, Merck, Pfizer, and Vanda Pharmaceuticals; is on the advisory boards for Amgen and Adaptive Biotechnologies; and received honoraria from Pfizer. His spouse is employed by and has stock options with Seattle Genetics. Damian J. Green receives research funding from Juno Therapeutics, a Celgene Company; Seattle Genetics; Cellectar Biosciences; and Janssen. He is a consultant to Celgene, GlaxoSmithKlein, and Seattle Genetics. Johnnie Orozco received research funding from Actinium Pharmaceuticals, Inc. John Pagel is a consultant for Pharmacyclics, Gilead, and Astra Zeneca. Brenda Sandmaier has research funding from Bellicum Pharmaceuticals and is a consultant for Actinium, Bristol-Meyers Squibb, and Kiadis. Her spouse is a consultant for AbbVie, AnaptysBio, Frazier Healthcare Ventures, Inipharm, and Oncoresponse; has equity ownership in AnaptysBio, Blaze Bioscience, EpiThany, Inipharm, Oncoresponse, and Mavupharma; and was formerly employed by Mavupharma. № other potential conflict of interest relevant to this article was reported.

\section{KEY POINTS}

QUESTION: Can treatment be performed with ${ }^{90}$ Y-anti-CD45 radioimmunotherapy to safely deliver the desired radiation doses without significant side effects?

PERTINENT FINDINGS: No dose limited toxicities occurred. The long biologic half-times observed using ${ }^{111}$ In-DOTA-BC8 for dosimetry estimates (approaching 10 days and longer) result in a more efficient delivery of radiation to the patient because decay takes place in vivo rather than after excretion.

IMPLICATIONS FOR PATIENT CARE: Radioimmunotherapy using preformed antibodies (such as that used in these 4 clinical trials-a preformed antibody against CD45 antigen) has potential in patients for whom other treatments for hematologic diseases failed. This treatment has the advantage of using passive immunotherapy (preformed antibodies) without the risk of activating the immune side effects that are seen with some of the active immunotherapies.

\section{REFERENCES}

1. Davies AJ. Radioimmunotherapy for B-cell lymphoma: $\mathrm{Y}^{90}$ ibritumomab tiuxetan and $\mathrm{I}^{131}$ tositumomab. Oncogene. 2007;26:3614-3628.

2. Kaminski MS, Estes J, Zasadny KR, et al. Radioimmunotherapy with iodine ${ }^{131} \mathrm{I}$ tositumomab for relapsed or refractory B-cell non-Hodgkin lymphoma: updated results and long-term follow-up of the University of Michigan experience. Blood. 2000;96:1259-1266.

3. Shadman M, Li H, Rimsza L, Leonard JP, et al. Continued excellent outcomes in previously untreated patients with follicular lymphoma after treatment with CHOP plus rituximab or CHOP plus ${ }^{131}$ I-tositumomab: long-term follow-up of phase III randomized study SWOG-S0016. J Clin Oncol. 2018;36:697-703.

4. Gopal AK, Press OW, Wilbur SM, Maloney DG, Pagel JM. Rituximab blocks binding of radiolabeled anti-CD20 antibodies (Ab) but not radiolabeled antiCD45-Ab. Blood. 2008;112:830-835.

5. Mawad R, Gooley TA, Rajendran JG, et al. Radiolabeled anti-CD45 antibody with reduced-intensity conditioning and allogeneic transplantation for younger patients with advanced acute myeloid leukemia or myelodysplastic syndrome. Biol Blood Marrow Transplant. 2014;20:1363-1368.

6. Mawad R, O'Donnell P, Gooley TA, et al. hematopoietic bone marrow transplantation (BMT) for patients with high-risk acute myeloid leukemia (AML), acute lymphoblastic leukemia (ALL), or myelodysplastic syndrome (MDS) using HLA-haploidentical related donors: a trial using radiolabeled anti-CD45 antibody combined with immunosuppression before and after BMT [abstract]. Blood. 2012;120:4164

7. Orozco JJ, Gooley T, Rajendran JG, et al. Anti-CD45 radioimmunotherapy followed by haploidentical allogeneic hematopoietic cell transplantation for advanced acute leukemia or high-risk MDS [abstract]. Blood. 2017;130(suppl 1):2048.

8. Cassaday RD, Press OW, Pagel JM, et al. safety and efficacy of escalating doses of ${ }^{90} \mathrm{Y}$ BC8-DOTA (anti-CD45) followed by carmustine, etoposide, cytarabine, and melphalan (BEAM) chemotherapy and autologous stem cell transplantation (ASCT) for high-risk lymphoma [abstract]. Biol Blood Marrow Transplant. 2018;24 (suppl):S251-S252.

9. Vo PT, Gooley T, Rajendran JG, et al. Safety and efficacy of yttrium-90-labeled anti-CD45 antibody ( ${ }^{90}$ Y-DOTA-BC8) followed by a standard reduced-intensity hematopoietic stem cell transplant (HCT) regimen for patients with refractory/ relapsed leukemia or high-risk myelodysplastic syndrome (MDS) [abstract]. Blood. 2018;132(suppl 1):1018.

10. Tuazon SA, Sandmaier BM, Orozco JJ, et al. A phase I trial of ${ }^{90}$ Y-BC8-DOTA (anti-CD45) monoclonal antibody in combination with fludarabine and TBI as conditioning for allogeneic peripheral blood stem cell transplant to treat high risk multiple myeloma [abstract]. Blood. 2017;130(suppl 1):910.

11. Matthews DC, Appelbaum FR, Eary JF, et al. Phase I study of ${ }^{131}$ I-anti-CD45 antibody plus cyclophosphamide and total body irradiation for advanced acute leukemia and myelodysplastic syndrome. Blood. 1999;94:1237-1247.

12. Cassaday RD, Press OW, Pagel JM, et al. Phase I study of a CD45-targeted antibody-radionuclide conjugate for high-risk lymphoma. Clinical Cancer Research. 2019;25:6932-6938.

13. Liang J, Yan D. Reducing uncertainties in volumetric image based deformable organ registration. Med Phys. 2003;30:2116-2122.

14. Rajendran JG, Fisher DR, Gopal AK, Durack LD, Press OW, Eary JF. High-dose ${ }^{131}$ I-tositumomab (anti-CD20) radioimmunotherapy for non-Hodgkin's lymphoma: adjusting radiation absorbed dose to actual organ volumes. J Nucl Med. 2004;45: 1059-1064.

15. Eary JF, Press OW, Badger CC, et al. Imaging and treatment of B-cell lymphoma. J Nucl Med. 1990;31:1257-1268.

16. Vallera DA, Elson M, Brechbiel MW, et al. Preclinical studies targeting normal and leukemic hematopoietic cells with yttrium-90-labeled anti-CD45 antibody in vitro and in vivo in nude mice. Cancer Biother Radiopharm. 2003;18:133-145.

17. Nemecek ER, Hamlin DK, Fisher DR, et al. Biodistribution of yttrium-90-labeled anti-CD45 antibody in a nonhuman primate model. Clin Cancer Res. 2005;11: 787-794.

18. Orozco JJ, Balkin ER, Gooley TA, et al. Anti-CD45 radioimmunotherapy with ${ }^{90} \mathrm{Y}$ but not ${ }^{177} \mathrm{Lu}$ is effective treatment in a syngeneic murine leukemia model. PLoS One. 2014;9:e113601.

19. Chinn PC, Leonard JE, Rosenberg J, Hanna N, Anderson DR. Preclinical evaluation of ${ }^{90}$ Y-labeled anti-CD20 monoclonal antibody for treatment of nonHodgkin's lymphoma. Int J Oncol. 1999;15:1017-1025.

20. Fisher DR, Shen S, Meredith RF. MIRD dose estimate report no. 20: radiation absorbed-dose estimates for ${ }^{111} \mathrm{In}$ - and ${ }^{90}$ Y-ibritumomab tiuxetan. J Nucl Med. 2009;50:644-652.

21. Stabin MG, Sparks RB, Crowe E. OLINDA/EXM: the second-generation personal computer software for internal dose assessment in nuclear medicine. $\mathrm{J} \mathrm{Nucl}$ Med. 2005;46:1023-1027.

22. Wiseman GA, Kornmehl E, Leigh B, et al. Radiation dosimetry results and safety correlations from ${ }^{90} \mathrm{Y}$-ibritumomab tiuxetan radioimmunotherapy for relapsed or refractory non-Hodgkin's lymphoma: combined data from 4 clinical trials. J Nucl Med. 2003;44:465-474.

23. Wiseman GA, Leigh BR, Dunn WL, Stabin MG, White CA. Additional radiation absorbed dose estimates for Zevalin radioimmunotherapy. Cancer Biother Radiopharm. 2003;18:253-258.

24. Matthews DC, Appelbaum FR, Eary JF, et al. Phase I study of ${ }^{131} \mathrm{I}$-anti-CD45 antibody plus cyclophosphamide and total body irradiation for advanced acute leukemia and myelodysplastic syndrome. Blood. 1999;94:1237-1247.

25. Pagel JM, Appelbaum FR, Eary JF, et al. ${ }^{131} \mathrm{I}$-anti-CD45 antibody plus busulfan and cyclophosphamide before allogeneic hematopoietic cell transplantation for treatment of acute myeloid leukemia in first remission. Blood. 2006;107:2184-2191.

26. Glatting G, Muller M, Koop B, et al. Anti-CD45 monoclonal antibody YAML568: a promising radioimmunoconjugate for targeted therapy of acute leukemia. J Nucl Med. 2006;47:1335-1341.

27. Matesan M, Fisher D, Wong R, et al. Radioimmunotherapy with ${ }^{90}$ Y-DOTA-BC8 (anti-CD45 antibody): effect of spleen size and uptake on bone marrow absorbed dose [abstract]. J Nucl Med. 2016;57(suppl 2):1018.

28. Aricò D, Grana CM, Vanazzi A, et al. The role of dosimetry in the high activity ${ }^{90}$ Y-ibritumomab tiuxetan regimens: two cases of abnormal biodistribution. Cancer Biother Radiopharm. 2009;24:271-275. 